\title{
Low Cost Fast Response Position Controller for Valve System in Automotive Application
}

\author{
Ohsuk Kwon ${ }^{\text {a }}$, Jungkyun Seo ${ }^{\text {a }}$, Jung-Hyo Lee ${ }^{\text {b,* }}$

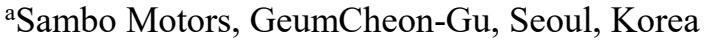

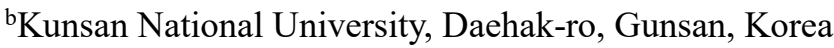 \\ *Corresponding Author: jhlee82@kunsan.ac.kr
}

\begin{abstract}
This paper shows the position control method for valve system in automotive application. Generally, automotive position control system has many restrictions such as cost and space, the mechanical structure of actuator implies high friction force. Besides, roughly designed mechanical system has comparatively high static friction and low coulomb friction, this difference makes deteriorating control performance. In this paper, low cost and fast response of position control method under the condition of high friction mechanical system. Proposed method is verified by comparing conventional control method using the experiment.
\end{abstract}

Keywords: Position control, Friction, Valve system.

\section{Introduction}

Recently, many mechanical components using in vehicle has been replaced by electrical components for high efficiency and eco-friendly. Not even hybrid electric vehicle or electric vehicle, MDPS(Motor Driven Power Steering) and ISG(Integrated Starter and Generator) are generally adopt in gasoline or diesel vehicle which are good examples of this change, and this change reaches the transmission system and engine valve system. However, general mechanical systems using in the valve, the allowable cost is very low and the space for implement is very narrow, therefore, the electrical system including actuator should have cost effective with small size. To achieve this, mechanical actuating system can not avoid to be roughly designed which implies high friction force. More worse, the difference between coulomb friction and static friction is very large, correct and fast response of position control is almost impossible to solve by conventional linear control system such as P, PI, or PID.

To regulate the position control against this friction torque, some research has been proposed. ${ }^{[1]-[5]}$ In [1], H infinite control and impulse control are combined for fast control response. Robust control is achieved by disturbance observer is proposed in [2]. Fuzzy controller [3] and neural network controller [4] are proposed to overcome this problem. In [5], adaptive control method for friction compensation is proposed. These methods can dramatically reduce the effect of the friction, however, the parameters have to be set are too much, and the processing burden for realization is also complex in low cost drive system.

This paper proposes the low cost position control method for high friction system. General control method for this position controller is P-PI control method which is described in [6]. Aforementioned, the correct and fast control can not be achieved under the mechanical system which has high friction condition with this linear controller. In this paper, comparing the performance of this conventional method and proposed control method by experiment.

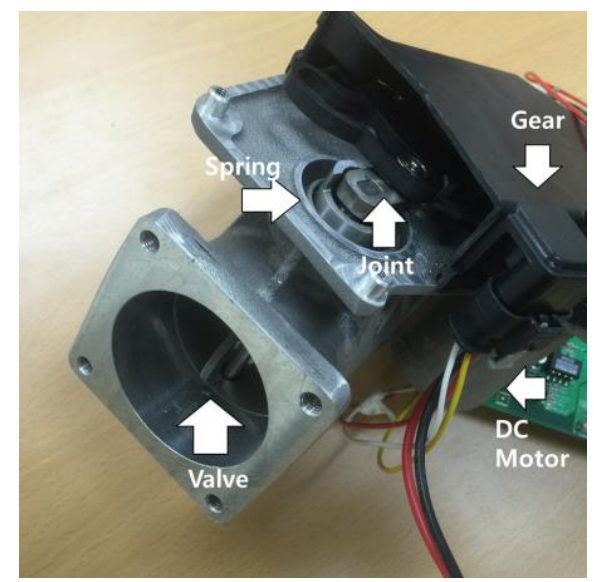

Fig. 1. Mechanical valve system 


\section{Modeling of Mechanical Valve System}

\subsection{Mechanical structure and its model}

Fig. 1 shows the control target of mechanical valve system. As shown on the figure, the actuator mechanic is composed of spring, throttle and gear. The torque from the motor applies to the spring, and pressed spring rotates the throttle to open or close the valve.

The motor to operate this system is selected as DC motor, then, the generated torque from the motor is below.

$$
T_{e}=k_{t} i_{a}
$$

where, $k_{t}$ is torque constant, $i_{a}$ is armature current of DC motor.

The mechanical equation of the valve system shown in Fig. 1 can be described as following general equation.

$$
T_{e}=J \frac{d^{2} \theta_{r}}{d t^{2}}+T_{\text {fric }}+T_{\text {spring }}+T_{L}
$$

On the other hands, rotating angle is transferred to linear motion by mechanical joint and gear, this linear motion can be expressed as below.

$$
\begin{aligned}
& x=r\left\{\cos \left(\theta_{L 0}\right)-\cos \left(\theta_{L}+\theta_{L 0}\right)\right\} \\
& \theta_{L}=\frac{\theta_{r}}{n}
\end{aligned}
$$

where, $n$ is the ratio of the gear.

The spring force affected by linear motion is below equation.

$$
F_{\text {spring }}=k_{\text {spr }}\left(x+x_{0}\right)
$$

Exchange this spring force to the spring torque, above equation can be transferred to below equation.

$$
\begin{aligned}
& T_{\text {spring }} \\
& =\frac{r k_{s p r}}{n} \sin \left(\theta_{L}+\theta_{L 0}\right) \times \\
& {\left[r\left\{\cos \left(\theta_{L 0}\right)-\cos \left(\theta_{L}+\theta_{L 0}\right)\right\}+x_{0}\right]}
\end{aligned}
$$




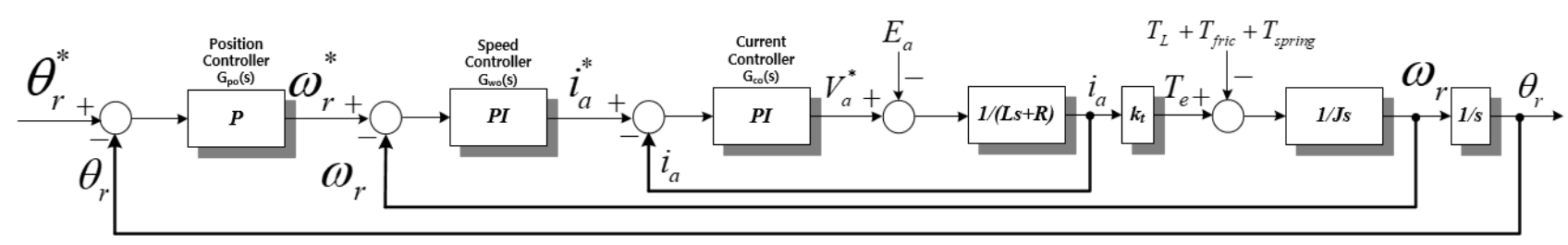

(a)

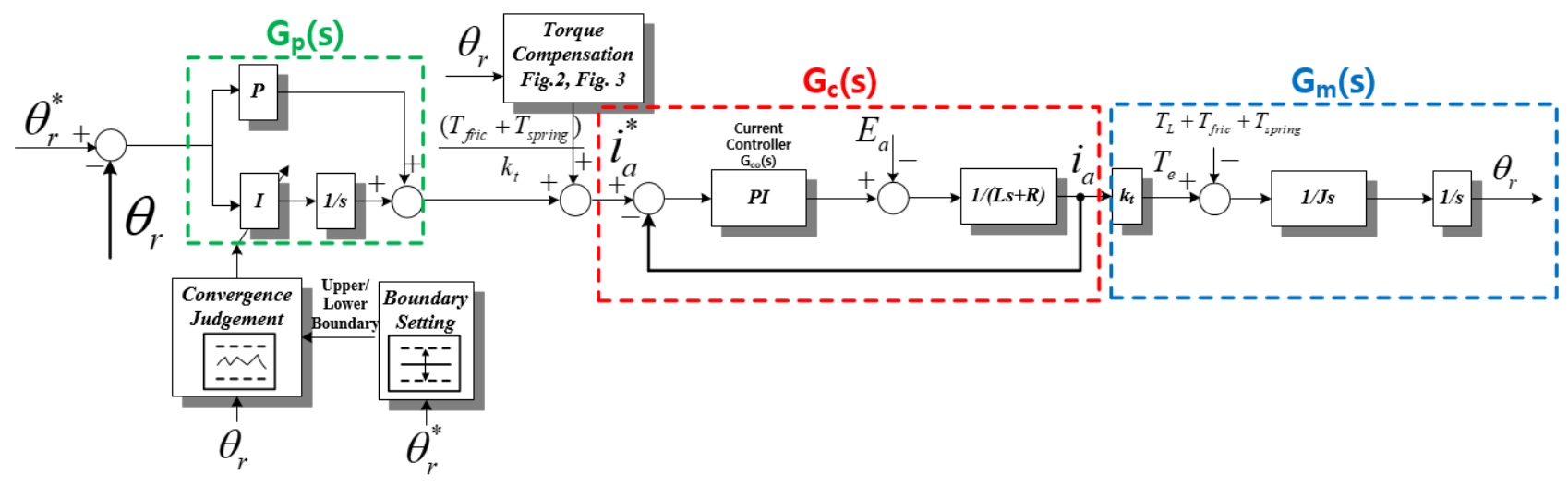

(b)

Fig. 4 Position control method (a) P-PI controller (b) Proposed controller

\subsection{Friction model of the gear and joint}

Adding the coulomb friction of the spring, the torque curve is shown on Fig. 2. However, as aforementioned, friction of this mechanical system has not only spring friction but also the gear friction. Due to the gear friction which contains backlash and slip, occurring nonlinear friction force which shape is static friction. In this paper, use the LuGre friction model which is described on [5].

$$
T_{\text {fric }}=\left[f_{c}+\left(f_{s}-f_{c}\right) e^{-\left(\omega_{r} / \omega_{s}\right)^{2}}\right] \operatorname{sgn}\left(\omega_{r}\right)
$$

Finally, adding the friction torque to total load model, it is deducted as follow equation.

$$
\begin{aligned}
& T_{e}=\frac{r k_{s p r}}{n} \sin \left(\theta_{L}+\theta_{L 0}\right) \times \\
& {\left[r\left\{\cos \left(\theta_{L 0}\right)-\cos \left(\theta_{L}+\theta_{L 0}\right)\right\}+x_{0}\right]} \\
& +\left\{f_{c}+\left(f_{s}-f_{c}\right) e^{-\left(\omega_{r} / \omega_{s}\right)^{2}}\right. \\
& \left.+T_{s p r_{-} c o l} \sin \left(\theta_{L}+\theta_{L 0}\right)\right\} \operatorname{sgn}\left(\omega_{r}\right)+T_{L}
\end{aligned}
$$

Fig. 3 shows the friction torque of the target valve system.

\section{Construction of the Position Controller}

Fig. 4 shows the conventional P-PI controller and proposed control system. As shown on the figure, proposed control system has no speed controller. The main reason to use no speed controller is the absence of suitable speed sensing device. Although the linear position sensor is adopt and the position information can be depicted from it, however, sensing dynamic of the position sensor is not enough to calculate motor speed. Moreover, the speed information is the derivative component of position information, it is essential to use the filter to mitigate the noise. This restricts the bandwidth of the controller more worse which is already restricted because of slow dynamic of the linear position sensor.

Due to the same reason, D controller can not be adopt because the effective derivative component of the position error is difficult to obtain. Moreover, it can amplify the noise of the position information signal. Therefore, the position controller is selected as PI controller in this system for low cost and high performance position control.

Firstly, position control transfer function described on Fig. 4 (b) can be expressed as follow.

$$
\frac{\theta_{r}}{\theta_{r}^{*}}=\frac{G_{p}(s) G_{c}(s) G_{m}(s)}{1+G_{p}(s) G_{c}(s) G_{m}(s)}
$$




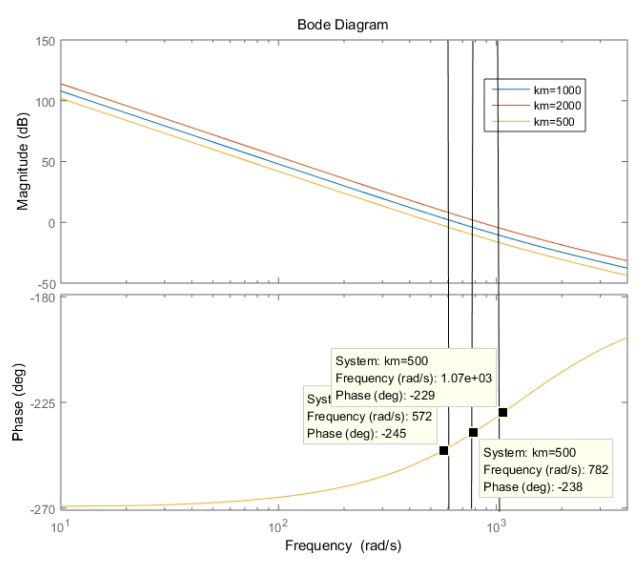

Fig. 5. Bode plots of controller

The control dynamic of the current controller is much faster than position controller, the transfer function of current controller can be approximated as 1 . Assuming that spring load torque and friction torque is fully compensated by feedforward path, the transfer function can be changed as follow.

$$
\frac{\theta_{r}}{\theta_{r}^{*}}=\frac{k_{p} k_{m} s+k_{i} k_{m}}{s^{3}+k_{p} k_{m} s+k_{i} k_{m}}
$$

where, $k_{m}$ is $k_{t} / J$.

As shown on the equation, the transfer function is expressed as third order characteristic equation. To obtain PI gains for stabilization, $k_{m}$ parameter is experimentally extracted. From that parameter value, then, verify PI gains under the condition from $0.5 k_{m}$ to $2 k_{m}$. The result is shown on Fig. 5. Note that if the $k_{m}$ parameter varied according to the linear position, the variable PI gain has to be adopt accordance with this position.

On the other hands, insert the transfer function to final value theorem, the error of step response can be obtained as below.

$$
e_{\infty}=\lim _{s \rightarrow 0} \frac{1}{1+G_{p}(s) G_{m}(s)}=1
$$

From the above equation, PI controller for position control has an error in steady state. To solve this problem, proposed control method set the allowable boundary to perceive that practical position follows the reference. If the practical linear position is going to inside of the boundary, the timer is activated to observe that the controlled position is stably located in the boundary or it is just transient operation.

Simultaneously, when it reaches the boundary, makes I control gain to zero accordance with the position error. Obviously, I controller integrates the position error to generates the current reference. However, integrating output from small position error is not effective under the high static friction torque condition. When the output reaches to enough for movement, the friction torque is radically changed to coulomb friction torque. This difference occurs the vibration of linear position. However, if the $\mathrm{P}$ controller is only operated in this range, this position vibration does not exist.

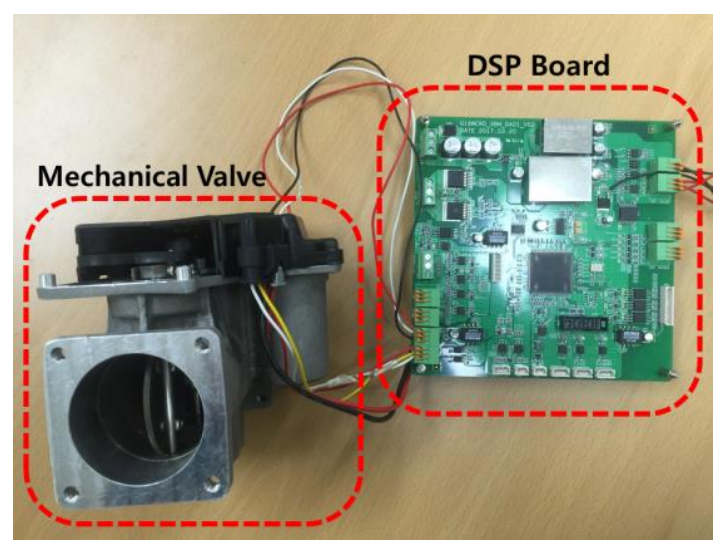

Fig. 6. Test setup

\section{Experiment}

Fig. 6 shows the test setup. To compare conventional control method, high performance DSP TMS320F28335 is used, and speed sensor is also instantaneously implemented in mechanical system. Sampling frequency of current controller and switching frequency are the same as $10[\mathrm{kHz}]$, obtained $k_{m}$ is 1000 which deducted from $k_{t}$ is 1 and $J$ is $0.001\left[\mathrm{kgm}^{2}\right]$.

Fig. 7 shows the experimental result using conventional P-PI controller. As shown on the figure, position is vibrates due to the large difference between static and coulomb friction torque. Until the current reaches $0.5[\mathrm{~A}]$ for forward direction, the position movement is very little because of static friction torque, however, above the $0.5[\mathrm{~A}]$ position is radically moved forward because of the sudden friction change to coulomb friction torque. The reverse operation is also similar to forward direction operation. As aforementioned, I controller integrates the small position error, and if the current reference is over the limit, the difference of static friction and coulomb friction torque causes the vibration.

Fig. 8 shows the experimental result of the proposed 
control method. The selected PI gains for stable operation which illustrated on chapter 3 , however, it is not suitable current reference to overcome static friction torque in initial operation. However, this problem can be solved by fine tuning of control gain by experiment. Although the starting delay, in contrast to conventional method, stable position control is achieved with allowable position error in steady state.

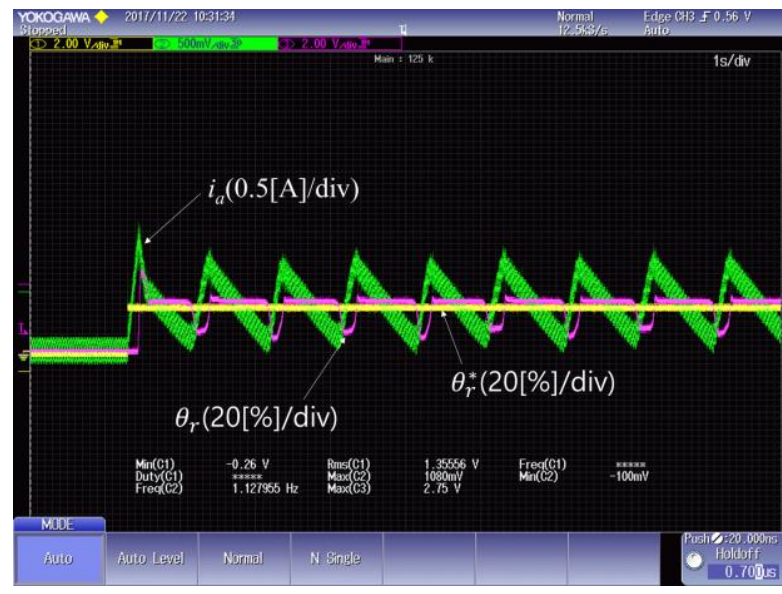

Fig. 7. Position control performance with P-PI controller

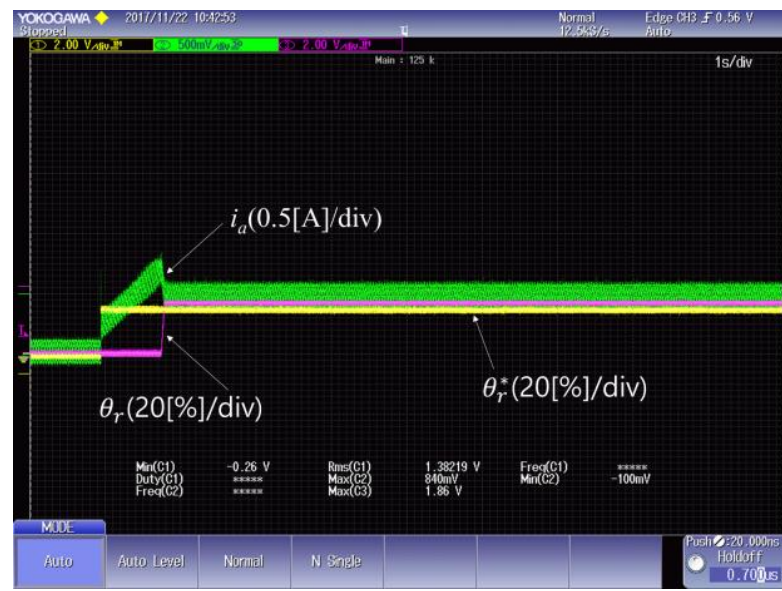

Fig. 8. Position control performance with proposed controller

\section{Conclusion}

This paper proposes the position control method for cost effective and fast response which can be used in vehicle valve system. Because the low cost mechanical system has the high difference of static friction and coulomb friction, the control performance is deteriorated with conventional linear controller. However, with the proposed control method, allowable boundary and selectable operation of I controller can achieves the proper control performance which has an acceptable error. Proposed method is verified by comparing conventional method in experiment.

\section{Acknowledgment}

This study has been conducted with World Class 300 R\&D business research funds, and thank the departments involved.

This work is supported the Human Resources Development Program (Grant No.20174010201350) by the Korea Institute of Energy Technology Evaluation and Planning (KETEP) grants.

\section{References}

(1) D.T. Kim, and Z. J. Zhang : "Position Control of a Pneumatic Cylinder Considering Friction Compensation", Journal of Korean Soc. Fluid Power Constr. Equip., Vol. 10, No. 1, pp. 1-6, 2013

(2) J.H. Byun : "A Study on the Position Control of a Motor Cylinder with Nonlineal Friction", Journal of the Korean Society for Power System Engineering, Vol. 12, No. 1, pp. 80-86, 2008

(3) Seema Chopra, Ranajit Mitra, Vijay Kumar: "Reduction of Fuzzy Rules and Membership Functions and Its Application to Fuzzy PI and PD Type Controllers", International Journal of Control Automation and Systems, Vol. 4, No. 4, pp. 438-447, 2006

(4) Zhisheng Ni, Mingyan Wang: "Research on the fuzzy neural network PID control of load simulator based on friction torque compensation", Sixth International Conference on Intelligent Human-Machine Systems and Cybernetics, pp. 292-295, 2014

(5) Jianyong Yao, Wenxiang Deng, and Zongxia Jiao: "Adaptive Control of Hydraulic Actuators With LuGre Model-Based Friction Compensation", IEEE Trans. on Ind. Elec., Vol. 62, No. 10, pp. 6469-6477, 2015

(6) Hyeong-Jin Kim, Hyung-Seok Park, Hong-Jun Heo, Jang-Mok Kim: "Improvement of Position Control Performance of EGR Valve System with Low Control Frequency", 2017 IEEE 3rd International Future Energy Electronics Conference and ECCE Asia (IFEEC 2017 ECCE Asia), pp. 394-399, 2017 